\title{
Erratum to: CE Separation and ICP-MS Detection of Gold Nanoparticles and Their Protein Conjugates
}

\author{
Joanna Legat ${ }^{1} \cdot$ Magdalena Matczuk $^{1} \cdot$ Federica Scaletti $^{2} \cdot$ Luigi Messori $^{2}$. \\ Andrei Timerbaev $^{3} \cdot$ Maciej Jarosz $^{1}$
}

Published online: 22 September 2017

(C) Springer-Verlag GmbH Germany 2017

\section{Erratum to: Chromatographia \\ DOI 10.1007/s10337-017-3387-y}

After publication of this work, we noted that we failed to include the complete list of all coauthors. The full list of authors has now been updated. The Authors' Competing interests and the Acknowledgement section modified accordingly. We are publishing this erratum to update the author list, which is as follows:

Joanna Legat (1), Magdalena Matczuk (1), Federica Scaletti (2), Luigi Messori (2), Andrei Timerbaev (3), Maciej Jarosz (1)

Acknowledgements Financial support from the National Science Centre, Poland (under Grant number 2015/17/B/ ST4/03707), Warsaw University of Technology and the Russian Foundation of Basic Research (Grant 16-03-00492) is gratefully acknowledged.

Conflict of interest Authors declare that they have no conflict of interest.

The online version of the original article can be found under doi:10.1007/s10337-017-3387-y.

Magdalena Matczuk

mmatczuk@ch.pw.edu.pl

1 Chair of Analytical Chemistry, Faculty of Chemistry, Warsaw University of Technology, Noakowskiego St. 3, 00-664 Warsaw, Poland

2 Department of Chemistry "Ugo Schiff", University of Florence, 50019 Florence, Italy

3 Vernadsky Institute of Geochemistry and Analytical Chemistry, Kosygin St. 19, 119991 Moscow, Russian Federation 